\title{
Modularity and cranial integration across ontogenetic stages in Martino's vole, Dinaromys bogdanovi
}

\author{
Tina Klenovšek ${ }^{1,3}$, Vida Jojić ${ }^{2}$ \\ ${ }^{1}$ Department of Biology, Faculty of Natural Sciences and Mathematics, University of Maribor, Koroška cesta 160, \\ 2000 Maribor, Slovenia \\ ${ }^{2}$ Department of Genetic Research, Institute for Biological Research 'Siniša Stanković, University of Belgrade, \\ Bulevar despota Stefana 142, 11060, Belgrade, Serbia \\ ${ }^{3}$ E-mail: tina.klenovsek@um.si
}

Key words: allometry, fluctuating asymmetry, geometric morphometrics, neurocranium, ontogeny, ventral cranium, viscerocranium

\begin{abstract}
We explored modularity and morphological integration of the ventral cranium during postnatal ontogeny in Martino's vole (Dinaromys bogdanovi). Two closely related phylogenetic groups, originating from the Central and Southeastern part of the species range in the western Balkans, were considered. As expected, both phylogroups had similar patterns of ontogenetic changes in cranial size and shape variation, modularity and integration. At the level of within individual variation, the hypothesis that the viscerocranial and neurocranial regions are separate modules was rejected, indicating that the hypothesized modules are not developmental, but rather functional. At the level of among individual variation, the viscerocranium and the neurocranium could not be recognized as separate modules at the juvenile stage. The strength of association between the hypothesized modules becomes lower with age which finally results in a clear 2-module organization of the ventral cranium at the adult stage. On the other hand, patterns of morphological integration for the cranium as a whole, the viscerocranium and the neurocranium stay consistent across ontogenetic stages. The developmental mechanism producing integration of the cranium as a whole, as well as integration of the neurocranium, varies throughout postnatal ontogeny. In contrast, we detected the ontogenetic stability of the mechanism responsible for covariation of viscerocranial traits which could provide ongoing flexibility of the viscerocranial covariance structure for high functional demands during lifetime. Findings from our study most likely support the idea of the 'palimpsest-like' model of covariance structure. Moreover, similarity or dissimilarity in the patterns of within and among individual variation in different sets of analyzed traits and comparisons across ontogenetic stages demonstrate how studies on small mammals other than mice can give new insights into postnatal cranial development.
\end{abstract}

\section{Contents}

Introduction

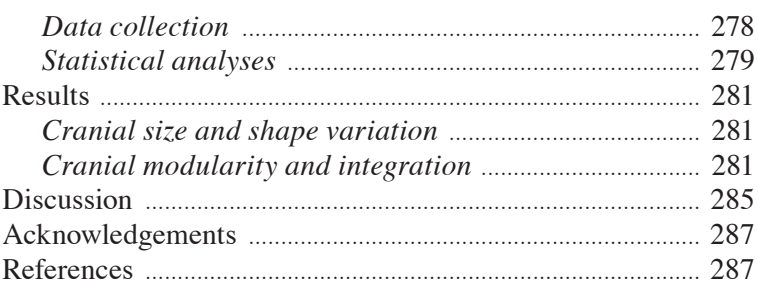

\section{Introduction}

Integration and modularity of organismal bodies and their parts are important aspects of morphological variability and popular research topics in developmental and evolutionary biology. The main idea is that to function as a whole, parts of organisms are integrated and vary jointly, but, because of different functions, developmental and genetic origins, integration is not evenly distributed but rather structured into modules (Olson and Miller, 1958; Cheverud, 1996a, b; Wagner, 1996; Wagner and Altenberg, 1996; Klingenberg et al., 2004). The concepts of morphological integration and modularity are therefore closely related (Klingenberg, 2008). Modules are units of complex systems that are internally tightly correlated and relatively independent from other modules or the remainder of the system, whereas integration refers to the patterns and strength of covariation within and among modules or within the system as a whole. Modular organization appears to be a general characteristic of biological systems and is present at all biological levels, from cells to whole organisms (Klingenberg et al., 2003). Moreover, by constraining or directing covariation among traits, modularity influences processes across a wide range 
of temporal scales, from individual development to macroevolution (Klingenberg, 2008; Sanger et al., 2012).

Modules can be identified also within skeletal structures, like the cranium and mandible, which have been most frequently studied in rodents, primates and other mammals (for review see Klingenberg, 2013). In rodents, most integration and modularity analyses have been performed on the mandible, which is a relatively simple structure with two well defined modules (Atchley et al., 1985; Cheverud et al., 1991, 1997; Leamy, 1993; Klingenberg et al., 2003, 2004; Jojić et al., 2007, 2012; Klingenberg, 2009). In the rodent cranium, patterns of modularity are less obvious and also inconsistent, which has been shown in different studies (Hallgrímsson et al., 2009; Jamniczky and Hallgrímsson, 2011; Jojić et al., 2011; Klingenberg, 2013). Nevertheless, the mammalian skull can be divided into two major functional components, the neurocranium composed of the braincase, eyes and ears, and the viscerocranium consisting of the jaw apparatus (Emerson and Bramble, 1993). In addition, the two main modules of the skull follow different growth patterns. The neurocranium grows rapidly until early postnatal life and completes most of its growth well before the viscerocranium, which follows a prolonged growth course (Moore, 1981). Craniofacial development has been thoroughly investigated in laboratory mice, also by direct morphometric studies of successive ontogenetic stages (e.g. Willmore et al., 2006; Zelditch et al., 2006). In these and other similar studies (e.g. Zelditch, 2005; Gonzalez et al., 2011; Klenovšek, 2014), it has been noted that in the rodent skull patterns of integration change over ontogeny. In contrast, integration and modularity studies on mammals from natural populations demonstrated stability of the covariance structures among populations within species (González-José et al., 2004), among closely related species (Marroig and Cheverud, 2001; Ackermann, 2005; Drake and Klingenberg, 2010; Singh et al., 2012), and even between distinct species (Hallgrímsson et al., 2004). Moreover, analysing morphological integration in the facial skeleton of humans, chimpanzees, bonobos and gorillas, Ackermann (2005) reported that these species show a similar pattern of integration during ontogeny. As emphasized by the same author, a similar pattern of ontogenetic integration shared among the hominoids suggests that common developmental/functional integrative processes may play an important role in keeping covariance structure stable across this lineage.
Depending on processes that can contribute to morphological covariation, four components of integration and modularity can be studied: functional, developmental, genetic and environmental (Klingenberg, 2014). Most studies of integration and modularity investigate how morphological variation is structured in the static context, i.e. among individuals within homogenous samples of a single population (or species) at a particular ontogenetic stage. However, these components can be studied in samples of multiple age stages within particular populations or species, i.e. at the ontogenetic level, as well as in samples of multiple species at any given ontogenetic stage, i.e. at the evolutionary level (Klingenberg, 2014). In complex morphological structures, such as the skull, patterns of integration are influenced by sequential and interacting developmental processes that can generate opposing effects and overwrite integration patterns produced by the previous effects (Hallgrímsson et al., 2009). Starting from the basic premise that morphological covariation results from two main developmental mechanisms (direct interactions between developmental pathways that produce the traits, or parallel variation of developmentally independent pathways that are simultaneously affected by external genetic or environmental factors), Klingenberg (2003, 2005) proposed the use of fluctuating asymmetry (FA) as a tool for studying developmental origin of morphological integration. Bilaterally symmetric structures in non-sessile organisms share the same genome and experience similar environmental conditions for left and right sides of the body. Thus, variation among individuals in their left-right asymmetry is mainly a result of random variation in developmental processes. It is now an established method to compare the patterns of covariation among traits of signed FA (variation within individuals), reflecting direct connections between developmental pathways only, with the patterns of covariation among individuals, which can be of both developmental origins. However, as far as we know, investigations on developmental mechanisms responsible for generation of covariation among traits have been conducted within morphological structures as a whole, but never within modules separately.

Allometry, the relationship between size and shape, can be a strong integrating factor (Zelditch, 1988; Hallgrímsson et al., 2006; Klingenberg, 2009), especially in ontogenetic data, where growth generates large size increases. Allometry may interfere with analyses of modularity as it usually affects all parts of the structure jointly (Klingenberg, 2009, 2013). With 
separation of size and shape data, geometric morphometrics offers a simple way of correcting shape data for the effect of allometry, and analyses of allometryfree data (Monteiro, 1999). Moreover, geometric morphometrics detects fine-scale morphological variability, which is essential for the studies of FA, and provides specialized and well established methodology for studies of integration and modularity. It is worth noting that geometric morphometric analyses based on 3D landmarks distributed across ventral, dorsal and lateral aspects of the skull represent the best choice, generally. However, in 2D geometric morphometric studies, the ventral surface of the cranium is very suitable for analyses due to its morphological organization, numerous anatomical landmarks (Ljubisavljević et al., 2010), and small error due to imaging and digitizing (Jojić et al., 2011) which is particularly important for the studies dealing with FA.

Rodent skulls are amongst the most investigated skeletal structures in terms of integration and modularity. Considering the vast number of rodent species, a very small fraction has been examined so far. In voles, a muroid rodent like mice, only the modularity of molars has been studied (Laffont et al., 2009; Labonne et al., 2014). A rare paleoendemic vole, Martino's vole, Dinaromys bogdanovi (Martino and Martino, 1922) is the only living member of the genus Dinaromys occupying a small, fragmented range in the western Balkans (Kryštufek and Bužan, 2008). Both mitochondrial (Kryštufek et al., 2007, 2012) and microsatellite (Bužan et al., 2010) DNA analyses revealed three allopatric phylogenetic groups (from the Northwestern, Central and Southeastern part of the species range) within the species, whereas geometric morphometric analyses of cranial shape variation (Kryštufek et al., 2012) retrieved significant differences among these phylogenetic groups. In general, voles are short-lived, proliferating rodents, which rarely survive for longer than two years. According to Arvicolinae standards, Martino's vole is in many ways exceptional (Kryštufek et al., 2000). Namely, it lives up to four years, has a slow reproduction rate (up to two litters a year) and reaches sexual maturity in the 2nd calendar year. Because D. bogdanovi is a longlived vole with well-defined age stages, this species is also a suitable organism for studying potential changes in the patterns of modularity and integration over postnatal ontogeny, a process that has been previously investigated in some other rodent skulls.

In this study we explored modularity and morphological integration of the ventral cranium across three ontogenetic stages (juveniles, subadults and adults) in two closely related phylogenetic groups of Martino's vole. According to the previous similar studies in small mammals (Willmore et al., 2006; Zelditch et al., 2006; Klenovšek, 2014), we expected to observe cranial size and shape variation over postanatal ontogeny, but also to detect ontogenetic change in its modularity and integration. Moreover, in the line with the study of Ackermann (2005), we assumed that two closely related phylogenetic lineages (from the Central and Southeastern part of the species range) would have similar pattern of these ontogenetic changes. Accordingly, we defined three objectives in this study. First, within each ontogenetic stage we tested the hypothesis that the ventral cranium comprises two modules, the viscerocranium and the neurocranium, and compared the strength of integration between the hypothesized modules over postnatal ontogeny. Second, we compared patterns of morphological integration for the cranium as a whole, the viscerocranium and the neurocranium across ontogenetic stages. Finally, we examined the developmental mechanism responsible for generation of covariance at a particular ontogenetic stage within the cranium as a whole, as well as within the hypothesized modules separately. By comparing the pattern of covariance in FA and that among individuals over ontogeny, we investigated which mechanism underlying morphological integration (direct developmental interactions or parallel variation in separate developmental pathways) is dominant at particular ontogenetic stages and whether it remains stable or varies throughout ontogeny. All analyses were performed simultaneously for allometry-included and allometry-corrected shape data.

\section{Material and methods}

\section{Samples}

We studied 215 skulls of Martino's vole (Table 1). All specimens were obtained from museum collections; 123 originated from wild populations and 92 from a captive colony. Wild specimens were caught in the area between the Neretva and Drim rivers, a range of the Central phylogenetic group of Martino's vole (Kryštufek et al., 2007, 2012), and are deposited in the Slovenian Museum of Natural History, Ljubljana, Slovenia; Department of Zoology, University of Zagreb, Zagreb, Croatia; and the National Museum of Bosnia and Herzegovina, Sarajevo, Bosnia and Herzegovina. 
Table 1. Sample sizes (n) of Dinaromys bogdanovi from two phylogenetic lineages.

\begin{tabular}{lll}
\hline Phylogenetic lineage & Age group & $\mathrm{n}$ \\
\hline Central (C) & Juveniles (0) & 28 \\
& Subadults (1) & 28 \\
& Adults (2) & 67 \\
Southeastern (SE) & Juveniles (0) & 33 \\
& Subadults (1) & 26 \\
& Adults (2) & 33 \\
\hline
\end{tabular}

Captive specimens comprised offspring of animals collected on Mt. Šara, within the range of the Southeastern phylogenetic group, and reared in the Natural History Museum, Belgrade, Serbia, between 1985 and 1991.

Laboratory-bred specimens had known ages of 6 days to 44 months. The age of wild specimens was assessed in studies of Kryštufek et al. $(2000,2012)$ by the length of the anterior root of the first lower molar measured on X-ray radiographs of the mandibles, as well as the trapping date and auxiliary traits such as body weight, skull size and shape. Besides root length, which is a good indicator of absolute age in Martino's vole (Kryštufek et al., 2010), survived winters can also be used, because in Martino's vole the reproductive period is not continuous (ibid., Petrov and Todorović, 1982). Individuals used in this study were categorized into three age groups: 0 - juveniles ( $\leq 4$ months old); 1 - subadults (5-12 months old, caught up to the end of the first winter); 2 - adults ( $>12$ months old, caught after the first survived winter). The $4^{\text {th }}$ month was used as a milestone between juveniles and subadults, because at that age Martino's voles start developing roots at the first lower molar (Kryštufek et al., 2000), and, according to Petrov and Todorović (1982), reach sexual maturity. However, according to Kryštufek et al. (2000) and Kryštufek and Bužan (2008) sexual maturity is attained after the first survived winter, which was used as the milestone between subadults and adults. All age groups were well represented and, in laboratory-bred specimens, also continuously distributed (with 5-10 day intervals in juveniles, 10-50 day intervals in subadults, and $<100$ day intervals in adults under 36 months of age). However, the sample of wildcaught specimens did not include individuals younger than 0.8-1.0 month old, which is the approximate age at natal emergence (Petrov and Todorović, 1982).

A previous study of the ventral side of the skull of
Martino's vole (Kryštufek et al., 2012) did not detect sexual dimorphism either in size or shape of the ventral cranium. The sexes were therefore pooled in the current study. Also geographic samples of wild specimens were pooled because they all originated from a single range, i.e. the Central phylogenetic group (Kryštufek et al., 2007, 2012). For convenience, abbreviations for the phylogroups (C - Central and SE Southeastern) are used in most of the text.

\section{Data collection}

The ventral side of each skull was photographed with a digital camera by the same person under constant conditions, with the hard palate parallel to the photographic lens. Twenty-nine (13 paired and 3 median) two-dimensional landmarks were digitized and scaled using the tpsDig (Rohlf, 2013) and tpsUtil program (Rohlf, 2010) (Fig. 1A). To estimate measurement error for shape we performed Procrustes analysis of variance (ANOVA-Klingenberg and McIntyre, 1998; Klingenberg et al., 2002) for a subset of 62 Martino's voles (29\% of the total sample size). Two images of each cranium were taken, and each image was digitized twice. The mean squares for FA exceeded the error components due to imaging and digitizing by more than 3.3 -fold and 14.5 -fold, respectively, while the mean squares for individual variation exceeded the error components due to imaging and digitizing by more than 46.9-fold and 203.7-fold, respectively. Therefore, all subsequent analyses were based on a single image per cranium and a single digitizing.

The landmark coordinates of original and reflected configurations were aligned using Generalized Procrustes Analysis (Rohlf and Slice, 1990; Dryden and Mardia, 1998) to extract centroid size (CS) and Procrustes coordinates. For the cases where cranial regions were analysed separately, the ventral cranium was subdivided into viscerocranial and neurocranial subsets of landmarks (Fig. 1A) followed by separate Procrustes fits. In the analyses of the shapes of morphological structures with object symmetry, such as the cranium, the variation among individuals in the averages of original and reflected configurations constitutes the symmetric component of shape variation, while the variation within individuals in the landmark deviations of the original configuration from the average of the original and reflected configuration constitutes the asymmetric component of shape variation (Klingenberg et al., 2002). 


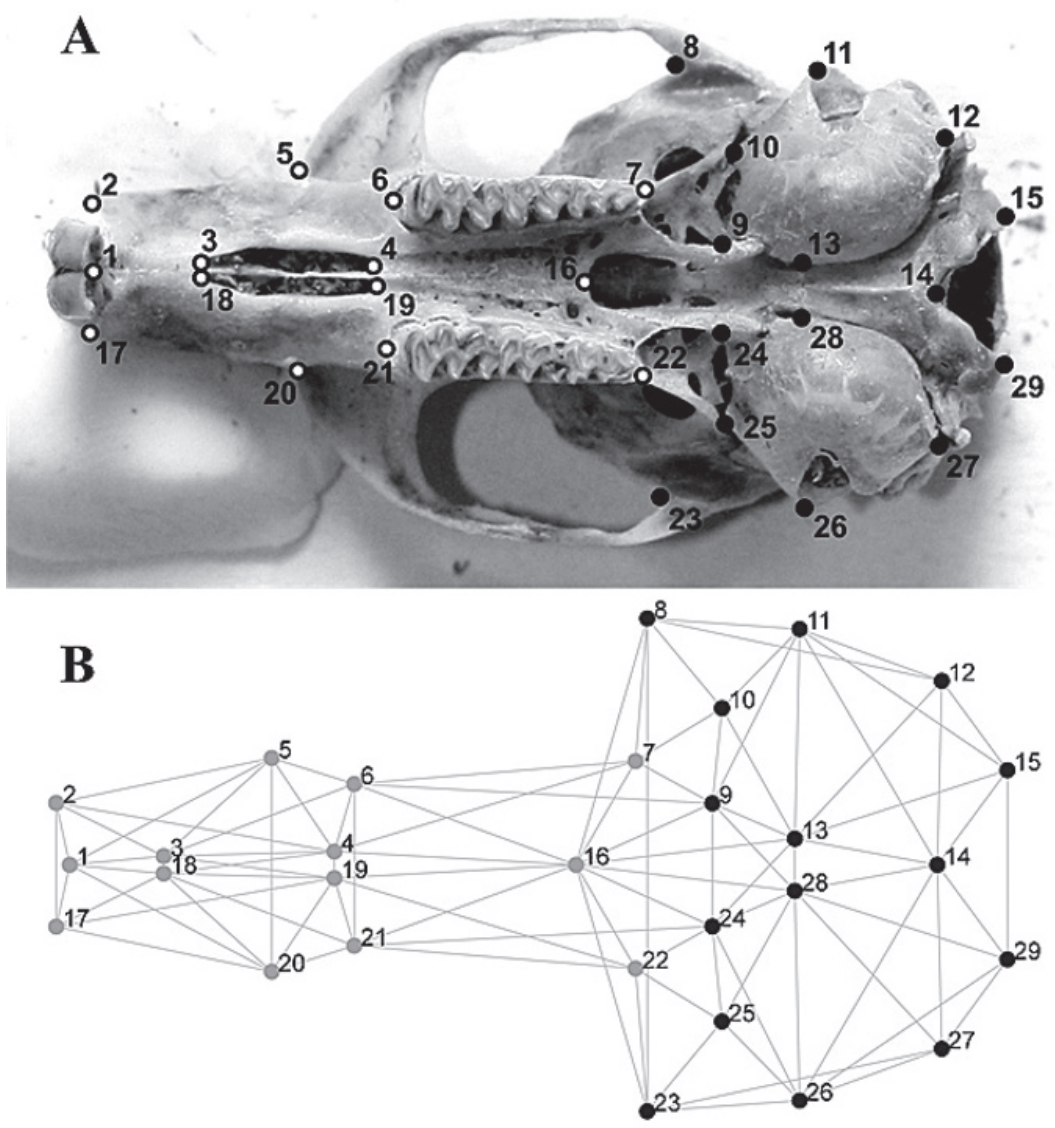

Fig. 1. A) Viscerocranial (white dots) and neurocranial (black dots) landmarks collected on the ventral surface of the cranium. B) Viscerocranial (grey dots) and neurocranial (black dots) landmarks connected by the edges of the adjacency graph.

\section{Statistical analyses}

We used a two-way ANOVA to test for size differences among phylogenetic groups, age categories, and their interactions, and a Tukey post-hoc honest significant difference (HSD) test for pairwise comparisons of the phylogenetic and age subgroups. Size variation was visualized with a graph of means showing the interaction between the effect of phylogenetic group and age category.

Shape variation was estimated using permutation tests (10 000 permutation rounds) for Procrustes distances among the phylogenetic and age subgroups, while shape relationships between the phylogenetic groups, as well as shape changes during postnatal ontogeny, were visualized using a graph of the first two canonical variates $(\mathrm{CV})$, and deformation grids based on the thin-plate spline (TPS) algorithm (Bookstein, 1991).

All the subsequent analyses were performed on phylogenetic and age subgroups separately.

To estimate the proportion of shape variation explained by size among (IND) and within (FA) indi- viduals, we performed multivariate regressions of the symmetric and asymmetric components of shape variation onto $\log \mathrm{CS}$, respectively. Covariance matrices generated from the symmetric and asymmetric components of shape variation, were used to investigate modularity and morphological integration including the effects of allometry, whereas covariance matrices of the residuals from these regressions were used to investigate modularity and morphological integration corrected for the effects of allometry.

To evaluate the hypothesis that in Martino's vole the anterior (viscerocranium) and the posterior (neurocranium) parts of the ventral cranium are modules, the configuration of 29 landmarks was divided into subsets of 14 (viscerocranium) and 15 (neurocranium) landmarks (Fig. 1A). The degree of covariation between the hypothesized modules was compared to all alternative spatially contiguous partitions with the same number of landmarks as in the hypothesized modules (Klingenberg, 2009). Spatial contiguity was obtained using an adjacency graph (Fig. 1B). The strength of association between the sets of landmarks 


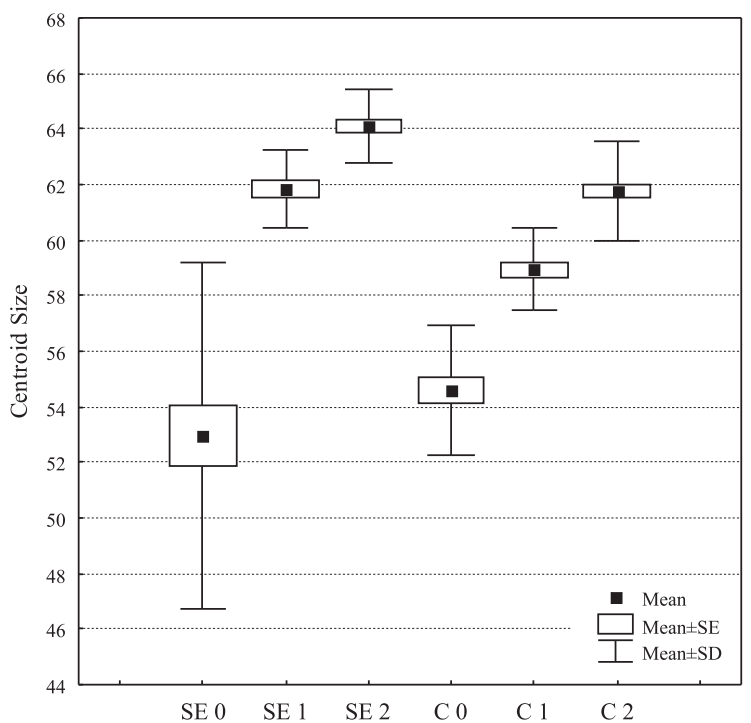

Fig. 2. Plot of centroid size means, standard deviations and standard errors for phylogenetic and age subgroups (SE 0 Southeastern juveniles; SE 1 - Southeastern subadults; SE 2 Southeastern adults; C 0 - Central juveniles; C 1 - Central subadults; C 2 - Central adults). was estimated with the Escoufier RV coefficients (Escoufier, 1973), which represented the amount of covariation scaled by the amount of variation within the two sets of variables. The RV coefficient takes the value of zero if the two sets of variables are completely uncorrelated and the value of one if the two sets of variables are completely interdependent. If the hypothesis of modularity holds, the RV coefficient for the selected partition should be the lowest value, or at least near the lower extreme of the distribution of RV coefficients of all alternative partitions (Klingenberg, 2009). To test for the difference in RV coefficients between all pairs of the phylogenetic and age subgroups, we performed a permutation test (10 000 iterations) under the null hypothesis of no difference in the RV coefficient (Fruciano et al., 2013).

We compared patterns of morphological integration at the level of individual variation for the cranium as a whole, the viscerocranium and the neurocranium, across ontogenetic stages, before and after correction for allometry. Matrix correlations between the covariance matrices generated from the symmetric compo-
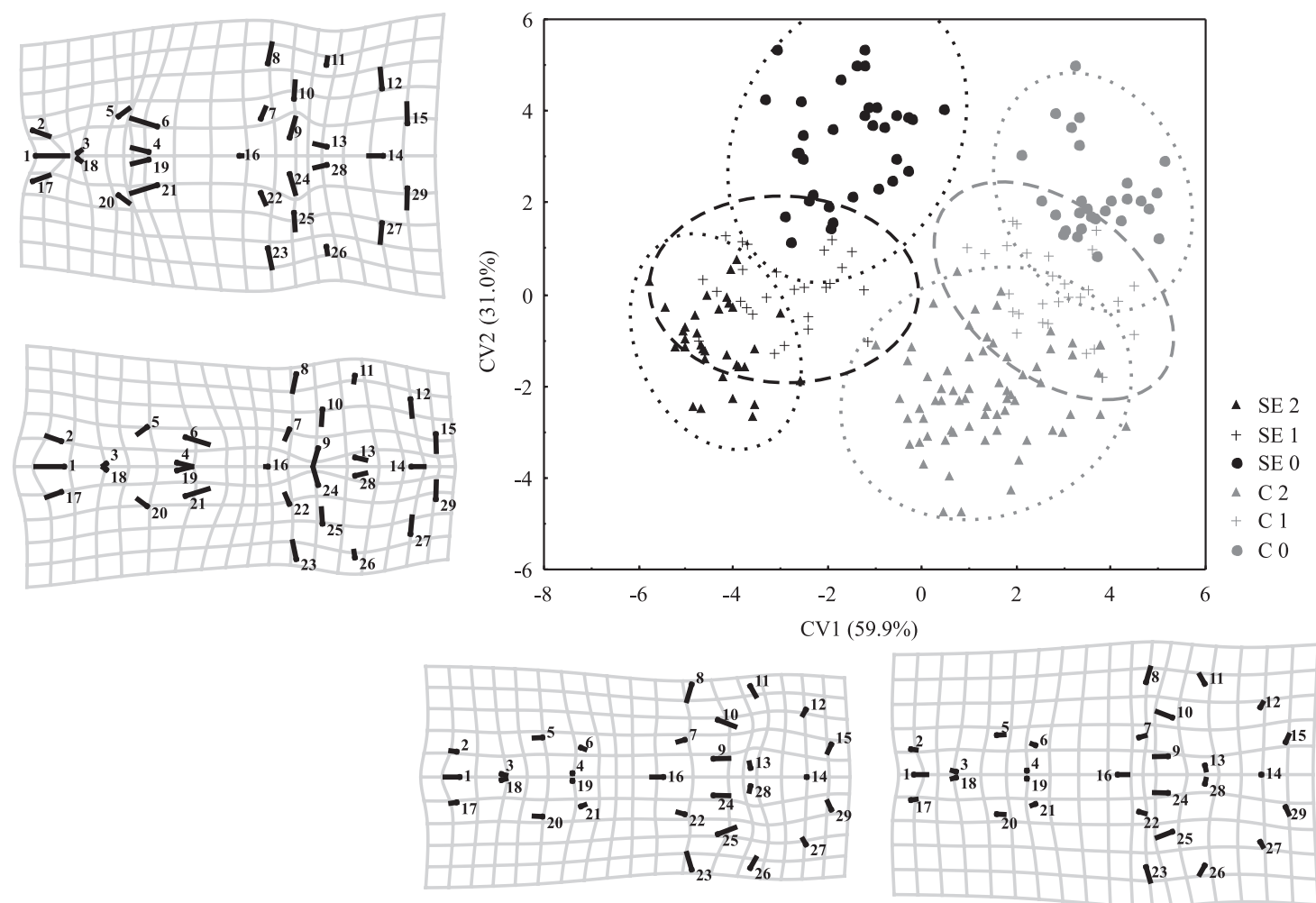

Fig. 3. Canonical variate analysis (CVA) for cranial shape differences among phylogenetic and age subgroups (SE 0 - Southeastern juveniles; SE 1 - Southeastern subadults; SE 2 - Southeastern adults; C 0 - Central juveniles; C 1 - Central subadults; C 2 - Central adults). Shape changes, magnified three times, are presented in the form of TPS deformation grids along the first (CV1) and second (CV2) axis. 
nent of shape variation were observed. The significance of these correlations was obtained using the matrix permutation test with 10000 iterations against the null hypothesis of complete dissimilarity between the respective covariance matrices (Cheverud et al., 1989), by permuting landmarks and including the diagonal entries of the matrices. However, the observed matrix correlations $\left(\mathrm{R}_{\mathrm{obs}}\right)$ are always estimated with error (Cheverud et al., 1989), and matrix repeatability has been suggested as a technique for estimating the impact of such error (Cheverud, 1996a; Marroig and Cheverud, 2001). To obtain adjusted matrix correlations $\left(\mathrm{R}_{\text {adj }}\right)$ between the respective matrices, their repeatabilities were calculated using the method of autocorrelation and 1000 bootstrap datasets.

Finally, the developmental mechanism underlying morphological integration for the cranium as a whole, the viscerocranium and the neurocranium, at a particular ontogenetic stage and its potential dynamics over postnatal ontogeny were examined by comparing the pattern of covariance in FA to the pattern of covariance among individuals within each age class. The significance of these correlations was also obtained using the matrix permutation test with 10000 iterations against the null hypothesis of complete dissimilarity between the respective covariance matrices (Cheverud et al., 1989).

Statistica v. 5.1 (StatSoft Inc., 1997), RVComparison 1.0 (Fruciano et al., 2013), and Mace (Marquez, 2014) were used for ANOVA, the permutation test for the difference in the RV coefficient, and matrix repeatabilities, respectively. All other analyses were performed using MorphoJ (Klingenberg, 2011).

\section{Results}

\section{Cranial size and shape variation}

As revealed by ANOVA, significant size differences were detected between the phylogenetic groups $\left(\mathrm{F}_{1,209}=\right.$ 8.27; $\mathrm{P}<0.01)$, among age categories $\left(\mathrm{F}_{2,209}=181.22 ; \mathrm{P}\right.$ $<0.0001)$, as well as for their interaction $\left(\mathrm{F}_{1,209}=11.10\right.$; $\mathrm{P}<0.0001$ ). Figure 2 gives a plot of centroid size means, standard deviations and standard errors for phylogenetic and age subgroups. The post hoc Tukey HSD test for unequal samples sizes showed statistically significant $(\mathrm{P}<0.05)$ size differences for all pairs of the analysed subgroups, except between the juveniles from the $\mathrm{SE}$ and $\mathrm{C}$ phylogenetic groups $(\mathrm{P}=0.2906)$, subadults and adults from the $\mathrm{SE}$ phylogenetic group $(\mathrm{P}=$
0.0581), and between subadults from the SE and adults from the $\mathrm{C}$ phylogenetic group $(\mathrm{P}=0.9999)$.

All pairwise mean shape comparisons of the phylogenetic and age subgroups were statistically significant $(\mathrm{P}<0.001)$. In both phylogenetic groups, the largest Procrustes distances were found between the juveniles and adults and the smallest between the subadults and adults. Canonical variate analysis for cranial shape differences among the analysed subgroups (Fig. 3) disclosed separation of SE and C phylogenetic groups along the first $\mathrm{CV}$ axis (59.9\% of variance), as well as discrimination of age categories along the second $\mathrm{CV}$ axis (31.0\% of variance). The group of $\mathrm{C}$ adults was the most variable along both $\mathrm{CV}$ axes. Compared to those from the $\mathrm{C}$ phylogenetic group (positive CV1 scores), animals from the SE phylogenetic group (negative CV1 scores) had crania compressed in the basicranial region with smaller auditory bulla and foramen magnum, constricted in the region of zygomatic arches, and somewhat elongated in the facial region. The most prominent ontogenetic shape changes, visible along the CV2 axis, also encompassed the basicranial, temporal and facial regions. In comparison to juveniles, adults of Martino's vole were characterized by slenderer crania in the region of zygomatic arches with an elongated and narrowed snout, relatively shorter palate, smaller auditory bulla and foramen magnum.

Multivariate regression of individual variation of shape onto $\log$ CS was statistically significant within each phylogenetic and age subgroup, except within SE adults $(\mathrm{P}=0.3322)$. The estimated effect of static allometry accounted for $58.27 \%(\mathrm{P}<0.0001), 9.02 \%$ ( $\mathrm{P}$ $=0043), 24.54 \%(\mathrm{P}<0.0001), 6.18 \%(\mathrm{P}=0.0396)$ and $11.77 \%(\mathrm{P}<0.0001)$ of the symmetric component of shape variation in SE juveniles, SE subadults, $C$ juveniles, $\mathrm{C}$ subadults and $\mathrm{C}$ adults, respectively. Regression of the signed asymmetries on log CS was not statistically significant $(\mathrm{P}>0.05)$ in each of the analysed phylogenetic and age subgroups.

\section{Cranial modularity and integration}

For the symmetric component of shape variation, the hypothesis of 2-module organization of the ventral cranium was confirmed in SE subadults and in SE and C adults (Fig. 4). In SE subadults, only 4 of 996 alternative partitions had RV coefficients that were lower than or equal to that observed for the partition into viscerocranium and neurocranium. In SE and C adults, only 13 and 1 out of 996 alternative partitions were with RV less than or equal to the a priori hypothesis, 
A
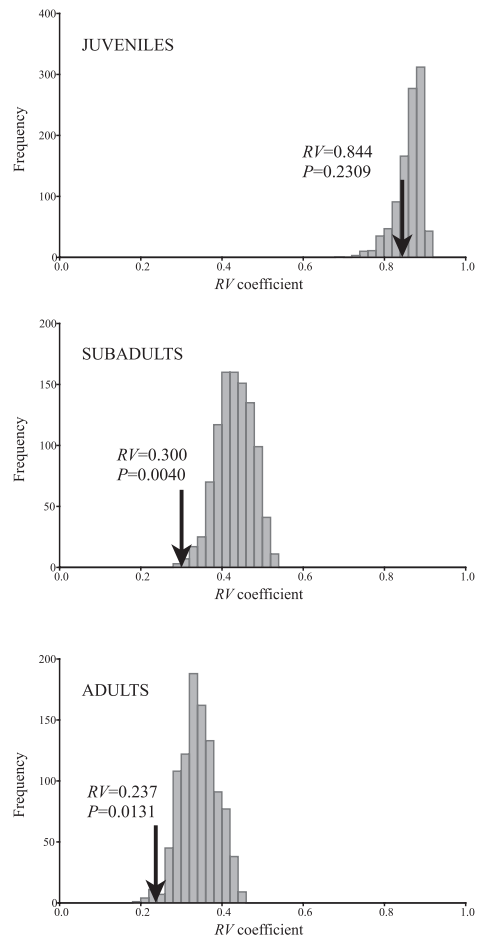

B
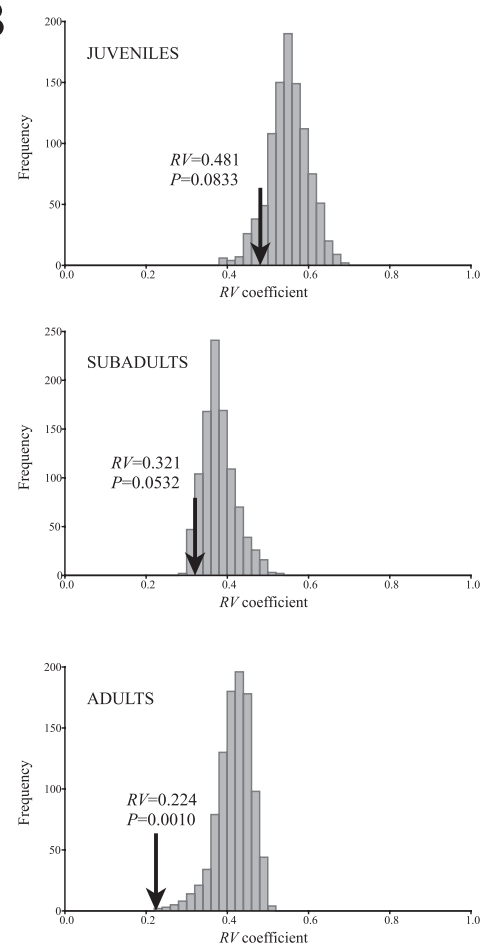

Fig. 4. Histograms of the RV coefficients for 996 alternative partitions of landmarks into spatially contiguous subsets for variation among individuals (including variation due to allometry) of juveniles, subadults and adults in A) Southeastern and B) Central phylogenetic group. The values of RV coefficients observed for the partition into viscerocranial and neurocranial regions and proportions $(\mathrm{P})$ of partitions with RV lower than or equal to the a priori hypothesis are indicated by arrows.

\begin{tabular}{|c|c|c|c|c|c|c|}
\hline & \multicolumn{3}{|c|}{ Allometry included } & \multicolumn{3}{|c|}{ Allometry corrected } \\
\hline & $\mathrm{RV}$ & $\begin{array}{l}\text { Difference } \\
\text { in } \mathrm{RV}\end{array}$ & $\mathrm{P}$ & RV & $\begin{array}{l}\text { Difference } \\
\text { in } \mathrm{RV}\end{array}$ & $\mathrm{P}$ \\
\hline SE 0 vs SE 1 & $\begin{array}{l}0.844 \\
0.300\end{array}$ & 0.543 & 0.0000 & $\begin{array}{l}0.257 \\
0.283\end{array}$ & 0.026 & n.s. \\
\hline SE 1 vs SE 2 & $\begin{array}{l}0.300 \\
0.237\end{array}$ & 0.064 & n.s. & $\begin{array}{l}0.283 \\
0.242\end{array}$ & 0.040 & n.s. \\
\hline SE 0 vs SE 2 & $\begin{array}{l}0.844 \\
0.237\end{array}$ & 0.607 & 0.0000 & $\begin{array}{l}0.257 \\
0.242\end{array}$ & 0.015 & n.s. \\
\hline $\mathrm{C} 0$ vs $\mathrm{C} 1$ & $\begin{array}{l}0.481 \\
0.321\end{array}$ & 0.161 & n.s. & $\begin{array}{l}0.328 \\
0.337\end{array}$ & 0.009 & n.s. \\
\hline $\mathrm{C} 1$ vs $\mathrm{C} 2$ & $\begin{array}{l}0.321 \\
0.224\end{array}$ & 0.096 & n.s. & $\begin{array}{l}0.337 \\
0.202\end{array}$ & 0.135 & n.s. \\
\hline $\mathrm{C} 0$ vs $\mathrm{C} 2$ & $\begin{array}{l}0.481 \\
0.224\end{array}$ & 0.257 & 0.0190 & $\begin{array}{l}0.328 \\
0.202\end{array}$ & 0.126 & n.s. \\
\hline
\end{tabular}

Table 2. RV coefficient observed for the partition into viscerocranial and neurocranial regions and differences in the RV coefficient between the analyzed subgroups (SE 0 - Southeastern juveniles; SE 1 - Southeastern subadults; SE 2 - Southeastern adults; C 0 - Central juveniles; C 1 - Central subadults; C 2 - Central adults), before and after correction for allometry. $P$ values are from permutation tests of the null hypothesis of no difference in the RV coefficient; n.s. not significant, $\mathrm{P}>0.05$. respectively. In $\mathrm{C}$ subadults, $\mathrm{RV}$ coefficient for the tested partition of landmarks was marginally significant $(\mathrm{P}=0.0532)$, while in juveniles, from both $\mathrm{SE}$ and $\mathrm{C}$ phylogenetic groups, the 2-module hypothesis was not supported. Additionally, in comparison to other analyzed phylogenetic and age subgroups, juveniles from the SE phylogenetic group were characterized by a limited range of rather high RV coefficients with the highest $\mathrm{RV}$ value for the subdivision of landmarks into viscerocranial and neurocranial regions. The permuta- tion test for the difference in RV coefficient observed for the partition into viscerocranial and neurocranial regions disclosed statistically significant differences in the strength of integration between the hypothesized modules for the following pairwise comparisons: SE juveniles and subadults, SE juveniles and adults, and C juveniles and adults (Table 2).

For the asymmetric component of shape variation, the RV coefficients between the viscerocranium and neurocranium and the proportions $(\mathrm{P})$ of partitions 

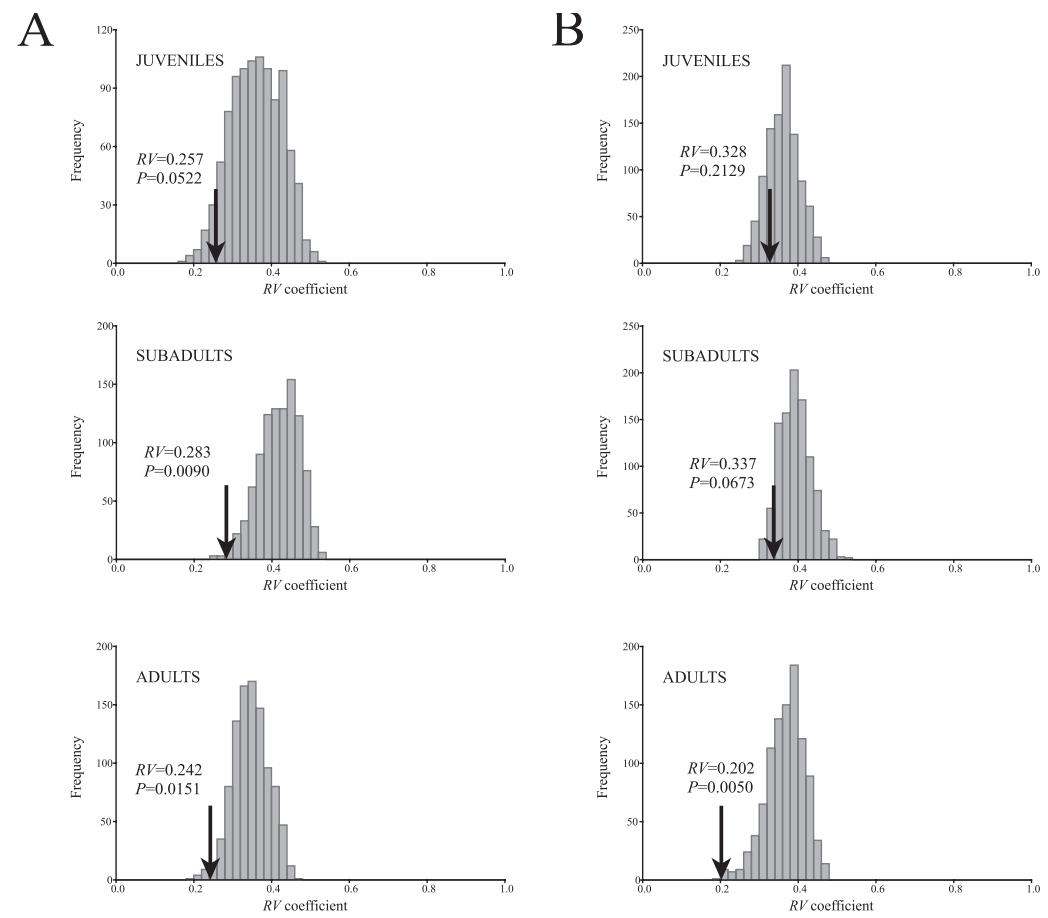

Fig. 5. Histograms of the RV coefficients for 996 alternative partitions of landmarks into spatially contiguous subsets for variation among individuals (corrected for the effect of allometry) of juveniles, subadults and adults in A) Southeastern and B) Central phylogenetic group. The values of RV coefficients observed for the partition into viscerocranial and neurocranial regions and proportions $(\mathrm{P})$ of partitions with $\mathrm{RV}$ lower than or equal to the a priori hypothesis are indicated by arrows. with RV lower than or equal to the a priori hypothesis were as follows: $\mathrm{RV}=0.398 ; \mathrm{P}=0.1526$ in $\mathrm{SE}$ juveniles, $\mathrm{RV}=0.479 ; \mathrm{P}=0.4167$ in $\mathrm{SE}$ subadults, $\mathrm{RV}=$ $0.338 ; \mathrm{P}=0.0894$ in $\mathrm{SE}$ adults, $\mathrm{RV}=0.401 ; \mathrm{P}=0.2339$ in $\mathrm{C}$ juveniles, $\mathrm{RV}=0.537 ; \mathrm{P}=0.7590$ in $\mathrm{C}$ subadults, and $\mathrm{RV}=0.421 ; \mathrm{P}=0.7139$ in $\mathrm{C}$ adults. Thus, at the level of FA, the hypothesis of 2-module organization of the ventral cranium was rejected in each of the analysed phylogenetic and age subgroups.

As shown in Fig. 5, when the allometric effects of size were removed from the symmetric component of shape variation, analyses of modularity revealed similar results. Again, the hypothesis of 2-module organization of the ventral cranium was confirmed in SE subadults and in SE and $\mathrm{C}$ adults. However, after correction for allometry, in the subgroup of SE juveniles, the RV coefficients for the alternative partitions and for the partition into viscerocranium and neurocranium dropped off drastically and their range became greater. Moreover, while at the level of individual variation, including variation influenced by allometry, 230 out of 996 alternative partitions had RV coefficients that were lower than or equal to that observed for the partition into viscerocranium and neurocranium $(\mathrm{P}=$ $0.2309)$, at the level of individual variation corrected for the effects of allometry, the RV coefficient for the tested partition of landmarks was marginally signifi- cant $(\mathrm{P}=0.0522)$ yielding results almost consistent with the hypothesis of modularity. The permutation test for the difference in the RV coefficient observed for the partition into viscerocranial and neurocranial regions after correction for allometry revealed no statistically significant differences in the strength of integration between the hypothesized modules for all pairwise comparisons (Table 2).

Covariance matrix comparisons across ontogenetic stages (Table 3) disclosed general similarity of the patterns of morphological integration for the cranium as a whole, as well as for the viscerocranial and neurocranial regions separately, in both SE and $\mathrm{C}$ phylogenetic groups. Moreover, observed similarity of the matrix covariance pattern for all pairwise comparisons was statistically highly significant $(\mathrm{P}<0.0001)$. Similar results were obtained after correction for allometry.

Table 4 shows comparisons of the pattern of covariance in fluctuating asymmetry (FA) to the pattern of covariance among individuals (IND) for the cranium as a whole, the viscerocranium and the neurocranium within phylogenetic and age subgroups. For the cranium as a whole, comparisons of these patterns disclosed their discrepancy within all phylogenetic and age subgroups except within $\mathrm{C}$ juveniles (before correction for allometry) and within juveniles from both SE and C phylogenetic groups (after correction for allometry). 


\begin{tabular}{lllllll}
\hline \multicolumn{5}{c}{ Allometry included } & & \multicolumn{3}{l}{ Allometry corrected } & \\
\hline whole cranium & $\mathrm{R}_{\text {obs }}$ & $\mathrm{R}_{\text {adj }}$ & $\mathrm{P}$ & $\mathrm{R}_{\text {obs }}$ & $\mathrm{R}_{\text {adj }}$ & $\mathrm{P}$ \\
\hline SE 0 vs SE 1 & 0.357 & 0.388 & $<0.0001$ & 0.485 & 0.558 & $<0.0001$ \\
SE 1 vs SE 2 & 0.691 & 0.777 & $<0.0001$ & 0.673 & 0.754 & $<0.0001$ \\
SE 0 vs SE 2 & 0.349 & 0.376 & $<0.0001$ & 0.540 & 0.615 & $<0.0001$ \\
C 0 vs C 1 & 0.569 & 0.647 & $<0.0001$ & 0.698 & 0.802 & $<0.0001$ \\
C 1 vs C 2 & 0.712 & 0.792 & $<0.0001$ & 0.742 & 0.826 & $<0.0001$ \\
C 0 vs C 2 & 0.588 & 0.638 & $<0.0001$ & 0.702 & 0.777 & $<0.0001$ \\
viscerocranium & & & & & & \\
SE 0 vs SE 1 & 0.507 & 0.550 & $<0.0001$ & 0.534 & 0.593 & 0.0195 \\
SE 1 vs SE 2 & 0.765 & 0.824 & $<0.0001$ & 0.769 & 0.826 & $<0.0001$ \\
SE 0 vs SE 2 & 0.594 & 0.636 & $<0.0001$ & 0.613 & 0.675 & 0.0015 \\
C 0 vs C 1 & 0.763 & 0.827 & $<0.0001$ & 0.829 & 0.899 & $<0.0001$ \\
C 1 vs C 2 & 0.837 & 0.892 & $<0.0001$ & 0.875 & 0.933 & $<0.0001$ \\
C 0 vs C 2 & 0.816 & 0.859 & $<0.0001$ & 0.835 & 0.883 & $<0.0001$ \\
neurocranium & & & & & & \\
SE 0 vs SE 1 & 0.435 & 0.459 & $<0.0001$ & 0.617 & 0.666 & $<0.0001$ \\
SE 1 vs SE 2 & 0.776 & 0.839 & $<0.0001$ & 0.743 & 0.802 & $<0.0001$ \\
SE 0 vs SE 2 & 0.501 & 0.525 & $<0.0001$ & 0.747 & 0.801 & $<0.0001$ \\
C 0 vs C 1 & 0.706 & 0.778 & $<0.0001$ & 0.793 & 0.888 & $<0.0001$ \\
C 1 vs C 2 & 0.742 & 0.808 & $<0.0001$ & 0.767 & 0.837 & $<0.0001$ \\
C 0 vs C 2 & 0.725 & 0.771 & $<0.0001$ & 0.855 & 0.922 & $<0.0001$ \\
& & & & & &
\end{tabular}

Table 3. Comparisons of the pattern of covariance among individuals (IND) for the cranium as a whole, the viscerocranium and the neurocranium between the analyzed subgroups (SE 0 - Southeastern juveniles; SE 1 - Southeastern subadults; SE 2 - Southeastern adults; C 0 - Central juveniles; C 1 - Central subadults; C 2 - Central adults), before and after correction for allometry. $\mathrm{R}_{\mathrm{obs}}-$ observed matrix correlations, $R_{\text {adj }}-$ adjusted matrix correlations. $\mathrm{P}$ values are from matrix permutation tests against the null hypothesis of complete dissimilarity between the respective covariance matrices.

Allometry included

\begin{tabular}{|c|c|c|c|c|}
\hline whole cranium & Correlation & $\mathrm{P}$ & Correlation & $\mathrm{P}$ \\
\hline SE 0 & 0.222 & n.s. & 0.442 & 0.0412 \\
\hline SE 1 & 0.395 & n.s. & 0.388 & n.s. \\
\hline SE 2 & 0.426 & n.s. & 0.421 & n.s. \\
\hline $\mathrm{CO}$ & 0.433 & 0.0311 & 0.495 & 0.0232 \\
\hline C 1 & 0.456 & n.s. & 0.448 & n.s. \\
\hline $\mathrm{C} 2$ & 0.407 & n.s. & 0.423 & n.s. \\
\hline \multicolumn{5}{|c|}{ viscerocranium } \\
\hline SE 0 & 0.390 & n.s. & 0.585 & n.s. \\
\hline SE 1 & 0.491 & n.s. & 0.455 & n.s. \\
\hline SE 2 & 0.370 & n.s. & 0.369 & n.s. \\
\hline $\mathrm{CO}$ & 0.536 & n.s. & 0.587 & n.s. \\
\hline C 1 & 0.506 & n.s. & 0.505 & n.s. \\
\hline $\mathrm{C} 2$ & 0.545 & n.s. & 0.547 & n.s. \\
\hline \multicolumn{5}{|l|}{ neurocranium } \\
\hline SE 0 & 0.448 & n.s. & 0.582 & n.s. \\
\hline SE 1 & 0.518 & n.s. & 0.526 & n.s. \\
\hline SE 2 & 0.649 & 0.0083 & 0.619 & 0.0212 \\
\hline $\mathrm{CO}$ & 0.523 & n.s. & 0.653 & 0.0277 \\
\hline C 1 & 0.586 & n.s. & 0.565 & n.s. \\
\hline C 2 & 0.672 & 0.0246 & 0.668 & 0.0266 \\
\hline
\end{tabular}

Table 4. Comparisons of the pattern of covariance in fluctuating asymmetry (FA) with the pattern of covariance among individuals (IND) for the cranium as a whole, the viscerocranium and the neurocranium within analyzed subgroups (SE 0 - Southeastern juveniles; SE 1 - Southeastern subadults; SE 2 Southeastern adults; C 0 - Central juveniles; C 1 - Central subadults; C 2 - Central adults), before and after correction for allometry. $\mathrm{P}$ values are from matrix permutation tests against the null hypothesis of complete dissimilarity between the respective covariance matrices; n.s. - not significant, $\mathrm{P}>0.05$.
These results indicate that, while in juveniles cranial trait covariation among individuals is primarily due to direct interactions between developmental pathways, in subadults and adults parallel variation of separate developmental pathways is the dominant mechanism responsible for generation of covariance. By compar- ing the pattern of covariance in FA and that among individuals for the viscerocranial region, we observed that these patterns were inconsistent within each of the analysed phylogenetic and age subgroups, demonstrating that integration of viscerocranial traits among individuals originates from parallel variation of separate 
developmental pathways. On the other hand, for the neurocranial region we found a difference between the patterns of FA and IND within all phylogenetic and age subgroups except within SE and C adults (before correction for allometry) and within $\mathrm{C}$ juveniles and adults from both SE and $\mathrm{C}$ phylogenetic groups (after correction for allometry), suggesting that in these subgroups integration of neurocranial traits among individuals is primarily due to direct interactions between developmental pathways. Besides these results, comparisons of the pattern of covariance in asymmetry and that among individuals over postnatal ontogeny yielded that, unlike the ontogenetic shift in mechanism underlying neurocranial integration and integration of the cranium as a whole, the mechanism underlying morphological integration in the viscerocranium (parallel variation in separate developmental pathways) remains stable throughout postnatal ontogeny.

\section{Discussion}

As expected, our study disclosed that two closely related phylogenetic groups of Martino's voles had similar pattern of cranial size and shape variation over postnatal ontogeny, as well as similar pattern of ontogenetic changes in cranial modularity and integration.

Analysis of variance revealed statistically significant effects of phylogenetic group, age category and their interaction on cranial size variation. Animals of older age categories (subadults and adults) from the SE phylogenetic group have larger crania than comparable age categories from the $\mathrm{C}$ phylogenetic group. Comparing size of the ventral cranium of wild-caught subadults and adults of Martino's vole, Kryštufek et al. (2012) found no significant differences among Northwestern, Central and Southeastern phylogenetic groups. The difference in cranial size between the wild-caught $\mathrm{C}$ and laboratory-bred SE Martino's voles observed herein is probably a consequence of the greater longevity of animals reared in laboratory conditions. Additionally, in contrast to $\mathrm{C}$ juveniles, the sample of laboratory-bred SE juveniles also included very young specimens which resulted in higher size variability and the proportion of size-related shape variance within this subgroup. On the other hand, shape changes between the wild-caught $\mathrm{C}$ and laboratory-bred SE Martino's voles (visible along CV1 axis) correspond in a one-to-one manner to shape differences between the $\mathrm{C}$ and SE wild-caught Martino's voles described previously by Kryštufek et al. (2012). Our analyses of cranial shape variation disclosed the largest Procrustes distances between the juveniles and adults, as well as ontogenetic changes in cranial shape visible along the CV2 axis. The same ontogenetic shape differences were present in both phylogenetic groups and are typical for postnatal skull development in tetrapods (Emerson and Bramble, 1993).

We found strong evidence for the 2-module organization of the ventral cranium at the level of the symmetric component of shape variation (individual variation) in both SE and C adults, as well as in subadults from the SE phylogenetic group. By contrast, at the level of the asymmetric component of shape variation (fluctuating asymmetry, FA), the hypothesis that the viscerocranial and neurocranial regions are separate modules was rejected in all phylogenetic and age subgroups. Since the covariation of FA mirrors integration and modularity of the processes involved in development of the analysed morphological structures (Klingenberg, 2003, 2005), our results indicate that the viscerocranium and the neurocranium of Martino's voles are probably functional, but not developmental modules. In a study of skull modularity of the European ground squirrel (Spermophilus citellus), Klenovšek (2014) revealed that the viscerocranium and the neurocranium are separate modules in both juveniles and adults, and that these modules were much more integrated with each other in juveniles than in adults indicating change in the strength of integration between the hypothesized modules during ontogeny. Our study also disclosed that for allometry-included shape data, the value of the RV coefficient between the viscerocranium and neurocranium declines from juveniles to older age categories in both phylogenetic groups. However, statistically significant differences in the strength of integration between the hypothesized modules were found between SE juveniles and subadults, between SE juveniles and adults, as well as between $\mathrm{C}$ juveniles and adults. For allometry-corrected shape data, no statistically significant difference in RV coefficient between the hypothesized modules across ontogeny was found. Accordingly, these results suggest that there is a contribution of size-related shape variation to the significant difference in the strength of integration between the viscerocranium and neurocranium found between younger and older age categories. In juveniles from the SE phylogenetic group, before the effect of allometry was corrected, all alternative partitions of landmarks had similar and high covariation. Similar covariation resulted in a limited range of 
$\mathrm{RV}$ coefficients in this subgroup, and this means that modularity is fairly weak (Drake and Klingenberg, 2010). In addition, high values for RV coefficient for alternative subdivisions, together with the rather high RV coefficient observed for the subdivision of landmarks into viscerocranial and neurocranial regions, point to generally tight connections among cranial traits. Static allometry accounted for the highest proportion of the symmetric component of shape variation in SE juveniles. Allometry can produce global integration throughout the whole morphological structure, counteracting modularity (Klingenberg, 2009). Therefore, failure to recognize the viscerocranium and the neurocranium as separate modules within the groups of SE juveniles, where the effect of size on cranial shape variation was most pronounced, was expected. The hypothesis of 2-module organization of the ventral cranium was almost confirmed in this group when the allometric effect was removed.

In both phylogenetic groups, the patterns of covariation in the symmetric component of shape variation were similar over postnatal ontogeny. Similarity in the covariance structure during postnatal ontogeny was detected for the cranium as a whole, the viscerocranium and the neurocranium, before, as well as after correction for allometry. Comparable to our study, Ackermann (2005) investigated patterns of covariation in the facial skeleton between adjacent developmental stages (infants, juveniles, adolescents, subadults and adults) within African apes (chimpanzees, bonobos and gorillas) and humans, and found a shared pattern of covariation among all ontogenetic stages within each species. Contrary to our findings, by examining the ontogenetic dynamics of covariance of skull shape in two rodent species, cotton rats (Sigmodon fulviventer Allen, 1889) and house mice (Mus musculus domesticus Rutty, 1772), Zelditch et al. (2006) detected a significant and large change in covariance structure from age to age. Willmore et al. (2006) also provided evidence that patterns of cranial covariation in mice change throughout postnatal growth. However, depending on the rodent species and study design, the age stages in different studies do not necessarily correspond, which certainly makes results from different studies difficult to compare. Moreover, most investigations dealing with comparisons of covariance structure across ontogenetic stages so far have involved laboratory animals. Other studies on mammals from natural populations revealed that covariance structures tend to be stable among populations within species (González-José et al., 2004), among closely related species (Marroig and Cheverud, 2001;
Ackermann, 2005; Drake and Klingenberg, 2010; Singh et al., 2012), and even between distinct species, such as mice and macaques (Hallgrímsson et al., 2004).

Comparisons of the pattern of covariance in FA to that among individuals revealed that the dominant mechanism responsible for generation of covariance among individuals depends on the sets of analysed traits, as well as on the ontogenetic stage. For the cranium as a whole at the juvenile stage, trait covariation is primarily due to direct interactions between developmental pathways, whereas at older ontogenetic stages parallel variation of separate developmental pathways is a dominant mechanism responsible for generation of covariance. The patterns of covariation of neurocranial traits among younger individuals (juveniles and subadults) result from parallel variation of separate developmental pathways, but among adults from direct interactions between developmental pathways. The leading mechanism responsible for generation of viscerocranial trait integration is parallel variation of separate developmental pathways, and this mechanism remains stable throughout postnatal ontogeny. These results confirmed previous considerations about "palimpsest-like" covariation structure (Hallgrímsson et al., 2009) referring to the modular nature of developmental processes themselves, i.e. to the processes acting at various stages of development that can have different patterns of integration and modularity and may involve differing sets of traits (Klingenberg, 2014). Furthermore, the ontogenetic stability of parallel variation in separate developmental pathways, as a developmental mechanism underlying integration of viscerocranial traits, may point to the following. Namely, according to Klingenberg (2004), if trait integration arises primarily by parallel variation of separate developmental pathways, the patterns of covariance will be more labile. Since the viscerocranium is characterized by a prolonged growth course (Moore, 1981) and a higher functional demand when compared to the neurocranium, the viscerocranial covariance structure is expected to be more flexible, and the mechanism providing this flexibility should be stable during postnatal ontogeny. The ontogenetic stability of the mechanism underlying the flexibility of the viscerocranial covariance structure is particularly important from the aspect of functional integration and modularity, because viscerocranial morphology is closely related to mastication, muscle-bone interactions and postnatal bone remodelling under mechanical loading.

In conclusion, similarities in pattern of ontogenetic changes in cranial modularity and integration observed 
between Central and Southeastern phylogenetic groups of Marino's voles could be summarized as follows. The viscerocranium and the neurocranium could not be recognized as separate modules at the juvenile stage. During postnatal ontogeny the strength of association between the hypothesized modules becomes lower, which finally results in a clear 2-module organization of the ventral cranium at the adult stage. On the other hand, patterns of morphological integration for the cranium as a whole, the viscerocranium and the neurocranium do not change during postnatal ontogeny. The developmental mechanism underlying integration of the cranium as a whole, as well as integration of the neurocranium, varies during postnatal ontogeny, whereas the mechanism responsible for generation of integration of viscerocranial traits remains stable. Phenotypic covariance structure at a particular ontogenetic stage reflects the cumulative effect of all developmental processes up to that stage. They may act at different times or overlap in time and space producing a "palimpsest-like" covariation structure (Hallgrímsson et al., 2009). As already mentioned, the failure to confirm the 2-module hypothesis for the covariation of FA suggests that the viscerocranium and the neurocranium of Martino's voles are not developmental modules. However, comparisons of the pattern of covariance in FA and that among individuals for the hypothesized modules separately, indicate the modularity of developmental processes themselves. Therefore, findings from our study most likely point to the palimpsest model of covariance structure, according to which the patterns of covariance caused by the successive developmental processes may partly overwrite each other and produce an integrating structure of non-modular nature, even though contributing developmental processes could be modular (Hallgrímsson et al., 2009; Klingenberg, 2014). Additionally, similarity or dissimilarity in the patterns of individual variation and FA in different sets of analysed traits and comparisons across ontogenetic stages demonstrate how studies on small mammals other than mice can give new insights into postnatal cranial development.

\section{Acknowledgements}

We thank Boris Kryštufek and Mojca Jernejc Kodrič of the Slovenian Museum of Natural History, Ljubljana; Milan Paunović and Jelena Jovanović of the Natural History Museum, Belgrade, for support and access to museum collections. We would also like to acknowledge the importance of the late D. Mirić's collection of laboratory-bred Martino's voles. Our gratitude is extended to C. Fruciano and F. Janžekovič for advice at various phases of the analysis, as well as to Marko Đurakić for helpful advice and suggestions on the early version of the manuscript. Also, we acknowledge two anonymous reviewers and the subject editor for all constructive comments and suggestions that have helped us to improve the current version of manuscript. The study was partly supported by the Ministry of Education, Science and Technological Development of Serbia, Grant No. 173003.

\section{References}

Ackermann RR. 2005. Ontogenetic integration of the hominoid face. Journal of Human Evolution 48: 175-197.

Atchley WR, Plummer AA, Riska B. 1985. Genetics of mandible form in the mouse. Genetics 111: 555-577.

Bookstein FL. 1991. Morphometric tools for landmark data. Cambridge: Cambridge University Press.

Bužan EV, Kryštufek B, Bryja J. 2010. Microsatellite markers confirm extensive population fragmentation of the endangered Balkan palaeoendemic Martino's vole (Dinaromys bogdanovi). Conservation Genetics 11: 1783-1794.

Cheverud JM. 1996a. Quantitative genetic analysis of cranial morphology in the cotton-top (Saguinus oedipus) and saddle-back (S. fuscicollis) tamarins. Journal of Evolutionary Biology 9: 5-42.

Cheverud JM. 1996b. Developmental integration and the evolution of pleiotropy. American Zoologist 36: 44-50.

Cheverud JM, Wagner GP, Dow MM. 1989. Methods for the comparative analysis of variation patterns. Systematic Zoology 38: 201-213.

Cheverud JM, Hartman SE, Richtsmeier JT, Atchley WR. 1991. A quantitative genetic analysis of localized morphology in mandibles of inbred mice using finite element scaling analysis. Journal of Craniofacial Genetics and Developmental Biology 11: 122-137.

Cheverud JM, Routman EJ, Irschick DJ. 1997. Pleiotropic effects of individual gene loci on mandibular morphology. Evolution 51: 2006-2016.

Drake AG, Klingenberg CP. 2010. Large-scale diversification of skull shape in domestic dogs: disparity and modularity. The American Naturalist 175: 289-301.

Dryden IL, Mardia KV. 1998. Statistical Shape Analysis. John Wiley and Sons, New York.

Emerson SB, Bramble DM. 1993. Scaling, allometry and skull design. Pp. 384-421 in: Hanken J, Hall BK, eds, The Skull. Functional and Evolutionary Mechanisms, vol. 3. Chicago: The University of Chicago Press.

Escoufier Y. 1973. Le traitement des variables vectorielles. Biometrics 29: 751-760.

Fruciano C, Franchini P, Meyer A. 2013. Resampling-based approaches to study variation in morphological modularity. PLOS ONE 8: e69376.

Gonzalez PN, Hallgrímsson B, Oyhenart EE. 2011. Developmental plasticity in covariance structure of the skull: effects of prenatal stress. Journal of Anatomy 218: 243-257.

González-José R, Van Der Molen S, González-Pérez E, Hernández M. 2004. Patterns of phenotypic covariation and correlation in modern humans as viewed from morphological integration. American Journal of Physical Anthropologynthropology 123: 69-77. 
Hallgrímsson B, Willmore K, Dorval C, Cooper DML. 2004. Craniofacial variability and modularity in macaques and mice. Journal of Experimental Zoology Part B: Molecular and Developmental Evolution 302: 207-225.

Hallgrímsson B, Brown JJY, Ford-Hutchinson AF, Sheets HD, Zelditch ML, Jirik FR. 2006. The brachymorph mouse and the developmental-genetic basis for canalization and morphological integration. Evolution and Development 8: 61-73.

Hallgrímsson B, Jamniczky H, Young NM, Rolian C, Parsons TE, Boughner JC, Marcucio RS. 2009. Deciphering the palimpsest: studying the relationship between morphological integration and phenotypic covariation. Evolutionary Biology 36: 355-376.

Jamniczky HA, Hallgrímsson B. 2011. Modularity in the skull and cranial vasculature of laboratory mice: implications for the evolution of complex phenotypes. Evolution and Development 13: 28-37.

Jojić V, Blagojević J, Ivanović A, Bugarski-Stanojević V, Vujošević M. 2007. Morphological integration of the mandible in yellow-necked field mice: The effects of B chromosomes. Journal of Mammalogy 88: 689-695.

Jojić V, Blagojević J, Vujošević M. 2011. B chromosomes and cranial variability in yellow-necked field mice (Apodemus flavicollis). Journal of Mammalogy 92: 396-406.

Jojić V, Blagojević J, Vujošević M. 2012. Two-module organization of the mandible in the yellow-necked mouse: a comparison between two different morphometric approaches. Journal of Evolutionary Biology 25: 2489-2500.

Klenovšek T. 2014. Skull modularity of the European ground squirrel Spermophilus citellus (Linnaeus, 1766). Acta Biologica Slovenica 57: 59-67.

Klingenberg CP. 2003. Developmental instability as a research tool: using patterns of fluctuating asymmetry to infer the developmental origins of morphological integration. Pp. 427-442 in: Polak M, ed, Developmental instability: causes and consequences. New York: Oxford University Press.

Klingenberg CP. 2004. Integration, modules, and development: molecules to morphology to evolution. Pp. 213-230 in: Pigliucci M, Preston K, eds, Phenotypic Integration: Studying the Ecology and Evolution of Complex Phenotypes. New York: Oxford University Press.

Klingenberg CP. 2005. Developmental constraints, modules and evolvability. Pp. 219-247 in: Hallgrímsson B, Hall BK, eds, Variation: A central concept in biology. Burlington, MA: Elsevier.

Klingenberg CP. 2008. Morphological integration and developmental modularity. Annual Review of Ecology, Evolution, and Systematics 39: 115-132.

Klingenberg CP. 2009. Morphometric integration and modularity in configurations of landmarks: tools for evaluating a priori hypotheses. Evolution and Development 11: 405-421.

Klingenberg CP. 2011. MorphoJ: an integrated software package for geometric morphometrics: Molecular Ecology Resources 11: 353-357.

Klingenberg CP. 2013. Cranial integration and modularity: insights into evolution and development from morphometric data. Hystrix, the Italian Journal of Mammalogy 24: 43-58.

Klingenberg CP. 2014. Studying morphological integration and modularity at multiple levels: concepts and analysis. Philosophical Transactions of the Royal Society B: Biological Sciences 369: 20130249.
Klingenberg CP, McIntyre GS. 1998. Geometric morphometrics of developmental instability: analyzing patterns of fluctuating asymmetry with Procrustes methods. Evolution 52: 1363-1375.

Klingenberg CP, Barluenga M, Meyer A. 2002. Shape analysis of symmetric structures: quantifying variation among individuals and asymmetry. Evolution 56: 1909-1920.

Klingenberg CP, Mebus K, Auffray J-C. 2003. Developmental integration in a complex morphological structure: how distinct are the modules in the mouse mandible? Evolution and Development 5: 522-531.

Klingenberg CP, Leamy LJ, Cheverud JM. 2004. Integration and modularity of quantitative trait locus effects on geometric shape in the mouse mandible. Genetics 166: 1909-1921.

Kryštufek B, Bužan E. 2008. Rarity and decline in palaeoendemic Martino's vole Dinaromys bogdanovi. Mammal Review 38: 267-284.

Kryštufek B, Kolarič K, Paunović M. 2000. Age determination and age structure in Martino's vole Dinaromys bogdanovi. Mammalia 64: 361-370.

Kryštufek B, Bužan EV, Hutchinson WF, Hänfling B. 2007. Phylogeography of the rare Balkan endemic Martino's vole, Dinaromys bogdanovi, reveals strong differentiation within the western Balkan Peninsula. Molecular Ecology 16: 12211232.

Kryštufek B, Engelberger S, Muzaferović Š, Bužan EV, Skok J, Škrijelj R, Herzig-Straschil B. 2010. Assessing population size of Martino's vole (Dinaromys bogdanovi) in central Bosnia. Hystrix, the Italian Journal of Mammalogy 21: 165169.

Kryštufek B, Klenovšek T, Bužan EV, Loy A, Janžekovič F. 2012. Cranial divergence among evolutionary lineages of Martino's vole, Dinaromys bogdanovi, a rare Balkan paleoendemic rodent. Journal of Mammalogy 93: 818-825.

Labonne G, Navarro N, Laffont R, Chateau-Smith C, Montuire S. 2014. Developmental integration in a functional unit: deciphering processes from adult dental morphology. Evolution and Development 16: 224-232.

Laffont R, Renvoisé E, Navarro N, Alibert P, Montuire S. 2009. Morphological modularity and assessment of developmental processes within the vole dental row (Microtus arvalis, Arvicolinae, Rodentia). Evolution and Development 11: 302-311.

Leamy L. 1993. Morphological integration of fluctuating asymmetry in the mouse mandible. Genetica $89: 139-153$.

Ljubisavljević K, Urošević A, Aleksić I, Ivanović A. 2010. Sexual dimorphism of skull shape in a lacertid lizard species (Podarcis spp., Dalmatolacerta sp., Dinarolacerta sp.) revealed by geometric morphometrics. Zoology 113: 168-174.

Marquez E. 2014. MACE. University of Michigan, Ann Arbor, MI. Available at http://www-personal.umich.edu/ emarquez /morph/. [accessed December 24, 2014]

Marroig G, Cheverud JM. 2001. A comparison of phenotypic variation and covariation patterns and the role of phylogeny, ecology, and ontogeny during cranial evolution of New World monkeys. Evolution 55: 2576-2600.

Martino V, Martino E. 1922. Note on a new snow vole from Montenegro. Annals and Magazine of Natural History 9: 413.

Monteiro LR. 1999. Multivariate regression models and geometric morphometrics: the search for causal factors in the analysis of shape. Systematic Biology 48: 192-199. 
Moore WJ. 1981. The Mammalian Skull. Cambridge, UK: Cambridge University Press.

Olson EC, Miller RL. 1958. Morphological Integration. Chicago, Illinois: University of Chicago Press.

Petrov BM, Todorović M. 1982. Dinaromys bogdanovi (V. et E. Martino, 1922). Pp. 193-208 in: Niethammer J, Krapp F, eds, Handbuch der Säugetiere Europas, Band 2/1. Wiesbaden: Akademische Verlagsgesellschaft.

Rohlf FJ. 2010. TpsUtil. Version 1.58. Department of Ecology and Evolution. State University of New York, Stony Brook, NY. Available at http://life.bio.sunysb.edu/morph/. [accessed November 10, 2014]

Rohlf FJ. 2013. TpsDig. Version 2.16. Department of Ecology and Evolution. State University of New York, Stony Brook, NY. Available at http://life.bio.sunysb.edu/morph/. [accessed January 25, 2012]

Rohlf FJ, Slice D. 1990. Extensions of the procrustes method for the optimal superimposition of landmarks. Systematic Zoology 39: 40-59.

Sanger TJ, Mahler DL, Abzhanov A, Losos JB. 2012. Roles for modularity and constraint in the evolution of cranial diversity among Anolis lizards. Evolution 66: 1525-1542.

Singh N, Harvati K, Hublin J-J, Klingenberg CP. 2012. Morphological evolution through integration: A quantitative study of cranial integration in Homo, Pan, Gorilla and Pongo. Journal of Human Evolution 62: 155-164.
StatSoft Inc. 1997. Statistica for Windows (Computer Program Manual). Tulsa. OK. USA.

Wagner GP. 1996. Homologues, natural kinds and the evolution of modularity. American Zoologist 36: 36-43.

Wagner GP, Altenberg L. 1996. Complex adaptations and the evolution of evolvability. Evolution 50: 967-976.

Willmore KE, Leamy L, Hallgrímsson B. 2006. Effects of developmental and functional interactions on mouse cranial variability through late ontogeny. Evolution and Development 8: 550-567.

Zelditch ML. 1988. Ontogenetic variation in patterns of phenotypic integration in the laboratory rat. Evolution 42: 28-41.

Zelditch ML. 2005. Developmental regulation of variability. Pp. 249-276 in: Hallgrímsson B, Hall BK, eds, Variation: A central concept in biology. Burlington, MA: Elsevier.

Zelditch ML, Mezey J, Sheets HD, Lundrigan BL, Garland TJ. 2006. Developmental regulation of skull morphology II: ontogenetic dynamics of covariance. Evolution and Development 8: 46-60.

Received: 13 March 2015

Revised and accepted: 5 January 2016

Published online: 28 July 2016

Editor: A. Ivanović 